\title{
Structural Dynamic Characteristics Test for a Three-story Building in Beijing
}

\author{
Jiemei MA \\ Institute of Geophysics \\ China Earthquake Administration \\ Beijing, China \\ e-mail: majm@cea-igp.cn \\ Ting YAN \\ Earthquake Administration of Beijing \\ China Earthquake Administration \\ Beijing, China \\ e-mail: yanting@bjseis.gov.cn
}

\author{
Xiandong KANG \\ Earthquake Administration of Beijing \\ China Earthquake Administration \\ Beijing, China \\ e-mail: kangxd@bjseis.gov.cn
}

\author{
Ying LIU \\ Earthquake Administration of Beijing, China Earthquake \\ Administration \\ Beijing, China \\ e-mail: liuying1975@bjseis.gov.cn
}

\begin{abstract}
Ambient dynamic test is applied to a 3-story building in Beijing and system identification is adopted to obtain the natural dynamic characteristics. The amplitudes of time histories recorded by different seismometers are compared. They increase with the floor number and two horizontal amplitudes are larger than the vertical one. Spectra analysis indicates that the spectra values of the recordings on the third floor are bigger than lower floors and Fourier spectra for vertical signals are relatively clearer and more remarkable than two horizontal recordings. Analysis suggests that ambient test is a useful tool for structural system identification.
\end{abstract}

Keywords-ambient test; fourier spectra; natural
characteristics; system identification

\section{INTRODUCTION}

Ambient test for a building is referred to the dynamic test when vibration of the building is excited by the environment. It is usually an effective method to identify the natural characteristics for structures and buildings. Ambient test is applied to the buildings with useful earthquake data recorded and dynamic characteristics identified from the recordings are used to compare with the information from the earthquake data (Kohler, etal, 2005). By compositing the natural characteristics obtained from system identification of ambient and earthquake, the actual measurements are introduced to update the numerical simulation models until they are consistent with real structures (Skolnik, 2005). Therefore, the ambient test is considered as the basis of dynamic load tests and carried out on various buildings and bridges (Ren, etal, 2004; Wang, etal, 2011; 2015).

\section{BUILDING PROFILE AND TEST ARRANGEMENT}

The building for test is located at Chaoyang District in Beijing. According to the Code for seismic design of buildings in China (GB 50011-2010), the seismic fortification intensity on its site is VIII and design basic earthquake acceleration value is $0.20 \mathrm{~g}$ ( $\mathrm{g}$ denotes acceleration of Gravity).It is a brick and concrete structure. The building has three floors, two over-ground floors and one underground floor, as shown in Figure 1. After communication with the owner, the final test plan was made based on the structural characteristics. An Etna Digital Recorder was respectively instrumented on geometric center of the third floor, the second floor and the first floor. It is a 3-channel recorder, with two horizontal sensors and one vertical sensor. The layout of the seismometers is shown in Figure 1 as well. For high accuracy, twice tests are recorded on the third floors. This instrument is a good cost-performance strong motion seismometers meeting the requirements of the broad seismic monitoring with excellent resolution in signal fidelity and data integrity. It also can be applied in seismic observation with large dynamic range.

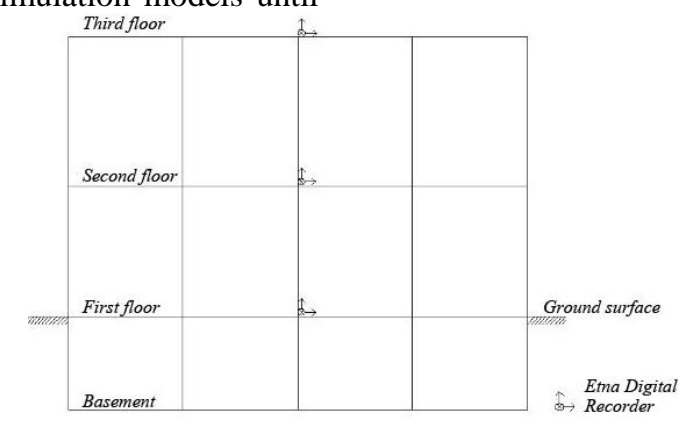

Figure 1. Building profile and layout of Etna digital recorders 


\section{SEGMENTED TEST RECORDINGS}

After in-situ ambient test, the time histories of the data recorded on different floors are shown in Fig.2. They respectively are time histories recorded on the first floor, the second floor, test 1 and test 2 on the third floor. The amplitudes enlarge with the increase of floor number.

These data are segmented and data of relatively smooth segment are selected to analyze identifying the dynamic characteristics of this building. To better demonstrate the different frequency components included in the building vibration, the fast Fourier transform is used to convert the signal into frequency domain signals. It is of more convenience to obtain the predominant frequency of the building. The Fourier spectrum plots are computed and they are indicated in Figure 3 and Figure 4. Among Figure 3, Fourier spectra in three directions including E-W (east-west), $\mathrm{N}-\mathrm{S}$ (north-south) and U-D (up-down) on the first, the second and the third floor are respectively illustrated in the upper, the middle and the bottom plots. As indicated in Figure 4, Fourier spectra in E-W, N-S and U-D on three floors are respectively listed in the upper, the middle and the bottom plots.
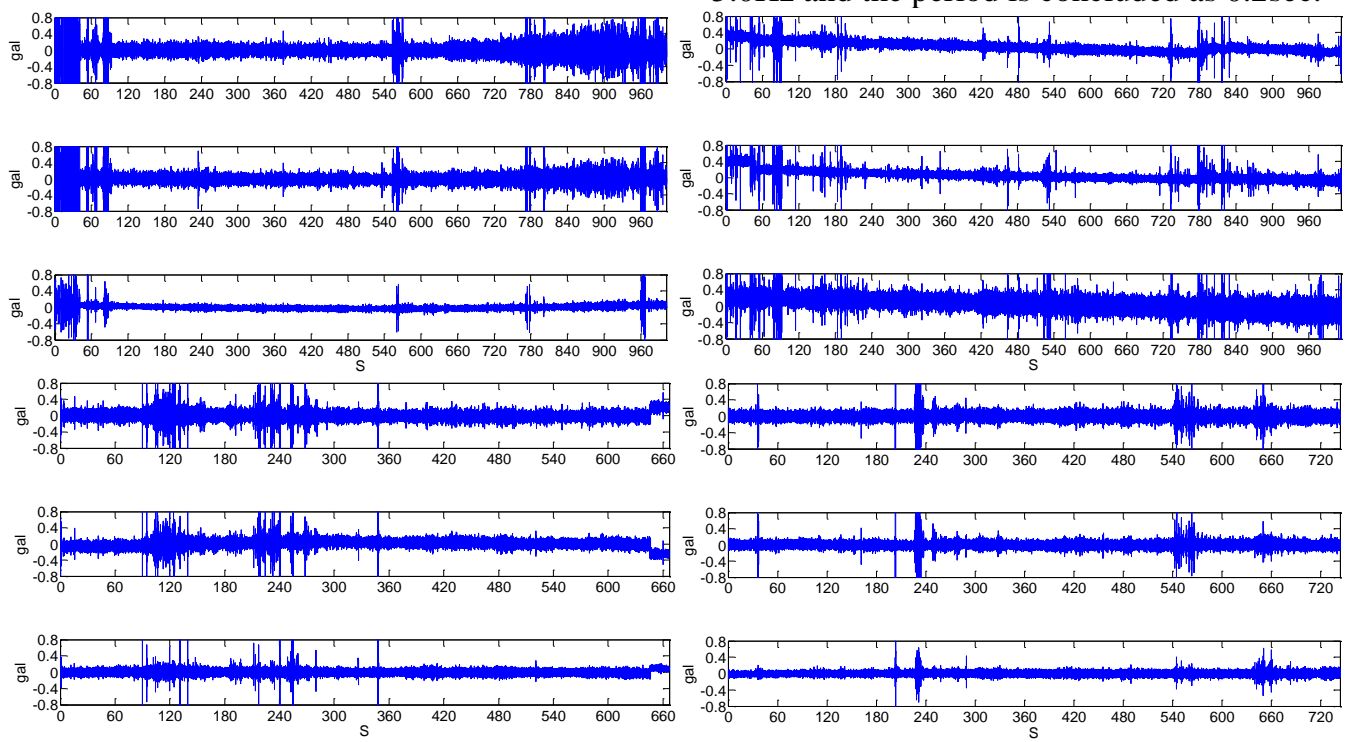

Figure 2.Three-channel time histories recorded on different floors (Upper left: First floor; Upper right: Second floor; Left bottom: Test 1 on the third floor; Right bottom: Test 1 on the third floor)
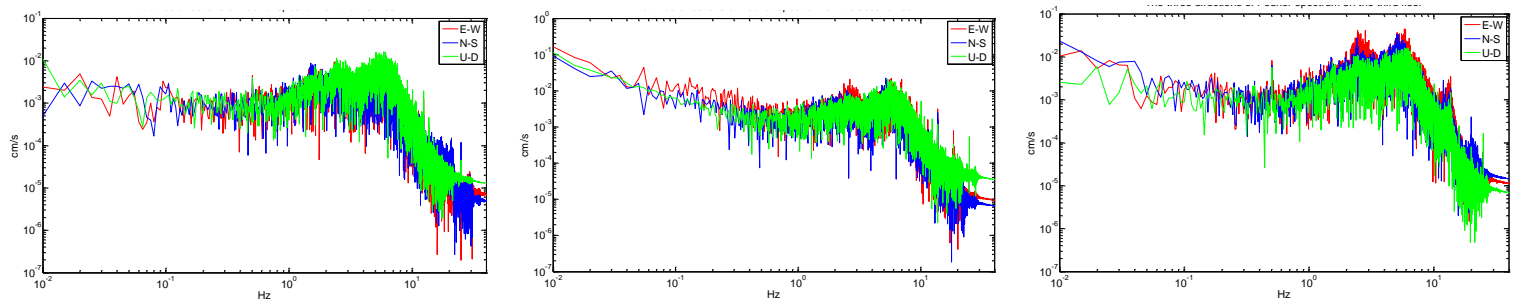

Figure 3. The Fourier spectrum plots in E-W, N-S and U-D directions on the first, the second and the third floor 

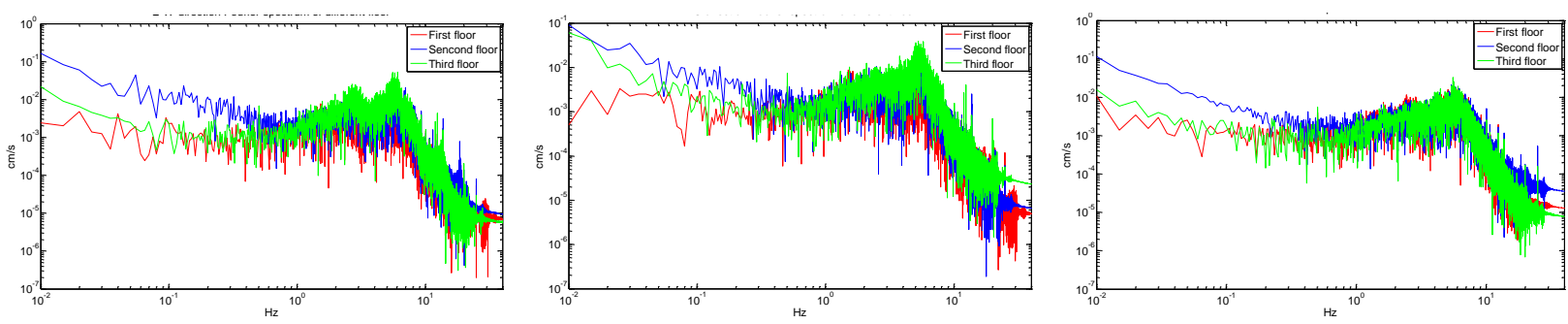

Figure 4. The Fourier spectrum plots in E-W, N-S and U-D directions on three floors
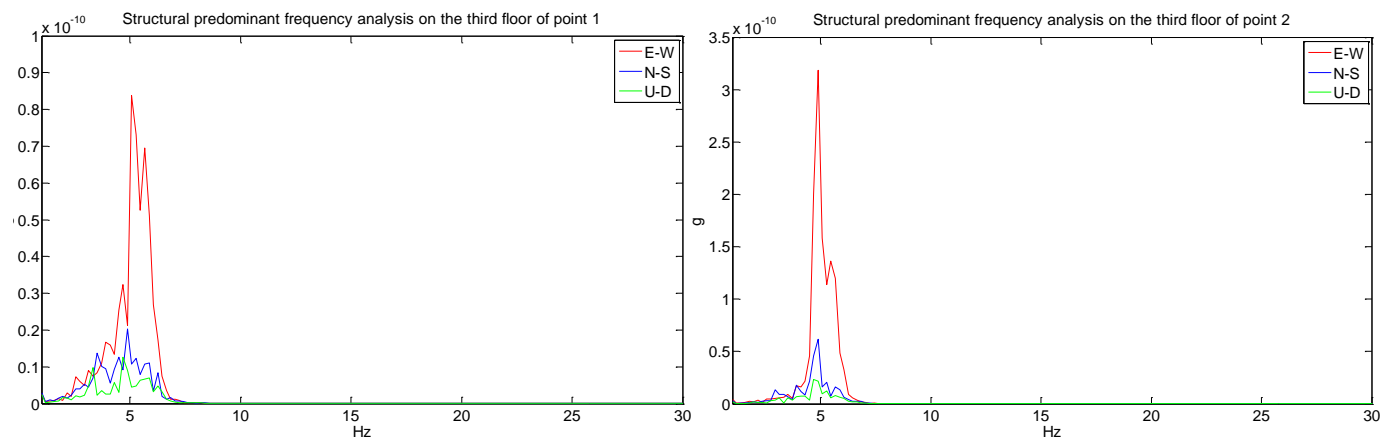

Figure 5. Fundamental frequency analysis based on Fourier spectra

\section{V.CONCLUSION}

Ambient test is carried out on a building in Beijing and natural dynamic characteristics are identified from the test recordings on different floors. Waveform analysis indicates that vibration amplitude increases with the increase of floor numbers. The amplitude in E-W direction is slightly larger than that in N-S direction and the amplitude in U-D direction is minimal. Spectrum analysis suggests that the fundamental natural period of this building is $0.2 \mathrm{sec}$, which is basically identical with the statistical values of the natural vibration period for three-floor buildings. This practice states that ambient test is an effective method for the dynamic characteristics identification of structures and buildings.

\section{ACKNOWLEDGEMENTS}

This paper is financially supported by the China National Special Fund for Earthquake Scientific Research in Public Interest (No.201408005) and also supported by Projects of
Science for Earthquake Resilience (No.XH16001 and No.XH17001).

\section{REFERENCES}

[1] Kohler, M. D., Davis, P. M., and Safak, E., Earthquake and ambient vibration monitoring of the steel frame UCLA Factor building [J]. Earthquake Spectra, 2005, 21(3): 715-736.

[2] Skolnik, D., Identification, Model Updating, and Response Prediction of the Louis Factor Building [D]. 2005. University ofCalifornia, Los Angeles.

[3] Ren Weixin,Blandford George, Harik Issam.Roebling Suspension Bridge.l:Finite-Element Model and Free Vibration Response. Journal of Bridge Engineering, 9(2):543-549.

[4] Wang Fei,Liu Bo, Yan Ting, LuoGuichun.Structural base-isolation analysis and micro-tremor test of an office building.Vibration and impact, 2011, 30(S):91-94.(In Chinese)

[5] Wang Fei, Liu Yinghua, Ren Zhilinand Tian Yuming. Structural Seismic Vibration Observation and Identificationfor the Building of Disaster Reduction Center in Beijing. Technology for Earthquake Disaster Prevention, 2015, 10(3): 539-546. (In Chinese) 